\title{
Robust Optimal Adaptive Control Method with Large Adaptive Gain
}

\author{
Nhan T. Nguyen* \\ NASA Ames Research Center, Moffett Field, CA 94035
}

\begin{abstract}
In the presence of large uncertainties, a control system needs to be able to adapt rapidly to regain performance. Fast adaptation is referred to the implementation of adaptive control with a large adaptive gain to reduce the tracking error rapidly. However, a large adaptive gain can lead to high-frequency oscillations which can adversely affect robustness of an adaptive control law. A new adaptive control modification is presented that can achieve robust adaptation with a large adaptive gain without incurring high-frequency oscillations as with the standard model-reference adaptive control. The modification is based on the minimization of the $\mathscr{L}_{2}$ norm of the tracking error, which is formulated as an optimal control problem. The optimality condition is used to derive the modification using the gradient method. The optimal control modification results in a stable adaptation and allows a large adaptive gain to be used for better tracking while providing sufficient stability robustness. Simulations were conducted for a damaged generic transport aircraft with both standard adaptive control and the adaptive optimal control modification technique. The results demonstrate the effectiveness of the proposed modification in tracking a reference model while maintaining a sufficient time delay margin.
\end{abstract}

\section{Introduction}

Adaptive control is a potentially promising technology that can improve performance and stability of a conventional fixed-gain controller. In recent years, adaptive control has been receiving a significant amount of attention. In aerospace applications, adaptive control has been demonstrated in a number of flight vehicles. For example, NASA has recently conducted a flight test of a neural net intelligent flight control system on board a modified F-15 test aircraft. ${ }^{1}$ The U.S. Air Force and Boeing have successfully developed and completed numerous flight tests of direct adaptive control on Joint Direct Attack Munitions (JDAM). ${ }^{2}$ The ability to accommodate system uncertainties and to improve fault tolerance of a control system is a major selling point of adaptive control since traditional gain-scheduling or fixed-gain control methods are viewed as being less capable of handling off-nominal operating conditions outside of a normal operating envelope. Nonetheless, these traditional control methods tend to be robust to disturbances and unmodeled dynamics when operated as intended.

In spite of the advances made in the field of adaptive control, there are several challenges related to the implementation of adaptive control technology in aerospace systems. These challenges include but are not limited to: 1) robustness in the presence of unmodeled dynamics ${ }^{3}$ and exogenous disturbances; 2 ) stability metrics of adaptive control as related to adaptive gain and input signals; 3 ) adaptation in the presence of actuator dynamic constraints; 4) on-line reconfiguration and control reallocation using non-traditional control effectors; and 5) time-scale separation in actuator systems with different time latency.

The absence of the verification and validation methods of adaptive control systems remain a major hurdle to the implementation of adaptive control in safety-critical systems. ${ }^{4,5}$ This hurdle can be traced to the lack of performance and stability metrics for adaptive control which poses a major challenge that prevents adaptive control from being implemented in safety critical systems. The development of verifiable metrics for adaptive control will be important in order to mature adaptive control technology for use in operational safety-critical systems. Of these, stability metrics of adaptive control are an important consideration for assessing system robustness to unmodeled dynamics and exogenous disturbances. In one aspect of verification and validation, a control system is usually certified by demonstrating that it meets an acceptable set of requirements or specifications for stability margins, among other things. Herein lies a major challenge for verification and validation as there is no existing standard tool for stability margin analysis of nonlinear adaptive control. The lack of stability metrics for adaptive control is viewed as a technology barrier to developing certifiable adaptive control for safety-critical systems. ${ }^{4,5}$

\footnotetext{
${ }^{*}$ Research Scientist, Intelligent Systems Division, Mail Stop 269-1, AIAA Associate Fellow
} 
Over the past several years, various model-reference adaptive control (MRAC) methods have been investigated. ${ }^{6-15}$ The majority of MRAC methods may be classified as direct, indirect, or a combination thereof. Indirect adaptive control methods are based on identification of unknown plant parameters and certainty-equivalence control schemes derived from the parameter estimates which are assumed to be their true values. ${ }^{16}$ Parameter identification techniques such as recursive least-squares and neural networks have been used in indirect adaptive control methods. ${ }^{7}$ In contrast, direct adaptive control methods directly adjust control parameters to account for system uncertainties without identifying unknown plant parameters explicitly. MRAC methods based on neural networks have been a topic of great research interest. ${ }^{8-10}$ Feedforward neural networks are capable of approximating a generic class of nonlinear functions on a compact domain within arbitrary tolerance, ${ }^{17}$ thus making them suitable for adaptive control applications. In particular, Rysdyk and Calise described a neural net direct adaptive control method for improving tracking performance based on a model inversion control architecture. ${ }^{8}$ This method is the basis for the intelligent flight control system that has been developed for the F-15 test aircraft by NASA. Johnson et al. introduced a pseudo-control hedging approach for dealing with control input characteristics such as actuator saturation, rate limit, and linear input dynamics. ${ }^{10}$ Hovakimyan et al. developed an output feedback adaptive control to address issues with parametric uncertainties and unmodeled dynamics. ${ }^{12}$ Cao and Hovakimyan developed an $\mathscr{L}_{1}$ adaptive control method to address high-gain control. ${ }^{13}$ Nguyen et al. developed a hybrid direct-indirect adaptive control to also deal with high-gain control issues during adaptation.

While adaptive control has been used with success in a number of applications, the possibility of high-gain control due to fast adaptation can be an issue. In certain applications, fast adaptation is needed in order to improve tracking performance when a system is subject to a large source of uncertainties such as structural damage to an aircraft that could cause large changes in system dynamics. In these situations, a large adaptive gains can be used in the adaptation in order to reduce the tracking error rapidly. However, there typically exists a balance between stability and adaptation. It is well known that high-gain control or fast adaptation can result in high-frequency oscillations which can excite unmodeled dynamics that could adversely affect the stability of an MRAC law. ${ }^{16}$ Recognizing this, some recent adaptive control methods have begun to address high-gain control, such as the $\mathscr{L}_{1}$ adaptive control ${ }^{13}$ and the hybrid direct-indirect adaptive control. ${ }^{14}$ In the former approach, the use of a low-pass filter effectively prevents any high frequency oscillation that may occur due to fast adaptation. In so doing, the reference model is no longer preserved and instead must be reconstructed using a predictor model. In the latter approach, direct and indirect adaptive control are blended together within the same control architecture. The indirect adaptive law is based on a recursive least-squares parameter estimation that is used to adjust parameters of a nominal controller to reduce the modeling error, and any remaining tracking error signal could then be handled by a direct adaptive law with a smaller adaptive gain.

Various modifications were developed to increase robustness of MRAC by adding damping to the adaptive law. Two well-known modifications in adaptive control are the $\sigma$-modification ${ }^{16}$ and $\varepsilon_{1}$ - modification. ${ }^{18}$ These modifications have been used extensively in adaptive control. This paper introduces a new adaptive law based on an optimal control formulation to minimize the $\mathscr{L}_{2}$ norm of the tracking error. The optimality condition results in a damping term proportional to the persistent excitation. The analysis shows that the adaptive optimal control modification can allow fast adaptation with a large adaptive gain without causing high-frequency oscillations and can provide improved stability robustness while preserving the tracking performance.

This paper is organized in two major sections. The first section is devoted entirely to the theoretical development of the new adaptive optimal control law which includes a Lyapunov stability proof and a derivation of an upper bound of the tracking error. The second section demonstrates a practical implementation of the optimal control modification in a neural net adaptive flight control architecture for a damaged generic transport aircraft. Simulation results are presented to demonstrate potential benefits of the new adaptive control law. 


\section{Theory of Optimal Control Modification}

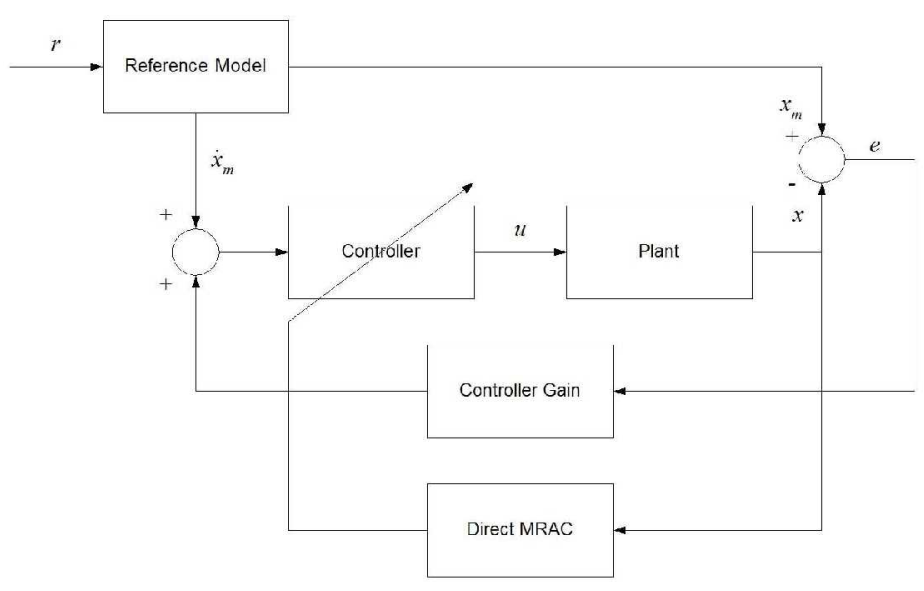

Fig. 1 - Direct MRAC

A direct MRAC problem is posed as follows:

Given a nonlinear plant as

$$
\dot{x}=A x+B[u+f(x)]
$$

where $x(t):[0, \infty) \rightarrow \mathbb{R}^{n}$ is a state vector, $u(t):[0, \infty) \rightarrow \mathbb{R}^{p}$ is a control vector, $A \in \mathbb{R}^{n \times n}$ and $B \in \mathbb{R}^{n \times p}$ are known such that the pair $(A, B)$ is controllable, and $f(x): \mathbb{R}^{n} \rightarrow \mathbb{R}^{p}$ is a bounded unstructured uncertainty.

Assumption 1: The uncertainty $f(x)$ can be approximated using a feedforward neural network in the form

$$
f(x)=\sum_{i=1}^{n} \theta_{i}^{*} \phi_{i}(x)+\varepsilon(x)=\Theta^{* \top} \Phi(x)+\varepsilon(x)
$$

where $\Theta^{*} \in \mathbb{R}^{m \times p}$ is an unknown constant weight matrix that represents a parametric uncertainty, $\Phi(x): \mathbb{R}^{n} \rightarrow \mathbb{R}^{m}$ is a vector of known bounded basis functions with Lipschitz nonlinearity, and $\varepsilon(x): \mathbb{R}^{n} \rightarrow \mathbb{R}^{p}$ is an approximation error.

Since $\Phi(x)$ is Lipschitz, then

$$
\left\|\Phi(x)-\Phi\left(x_{0}\right)\right\| \leq C\left\|x-x_{0}\right\|
$$

for some constant $C>0$, which implies a bounded partial derivative

$$
\left\|\frac{\partial \Phi(x)}{\partial x}\right\| \leq L
$$

for some constant $L>0$.

The set of basis functions $\Phi(x)$ is chosen such that the approximation error $\varepsilon(x)$ becomes small on a compact domain $x \in \mathbb{R}^{n}$. The universal approximation theorem for sigmoidal neural networks by Cybenko can be used for selecting a good set of basis functions $\Phi(x) .{ }^{17}$ Alternatively, the Micchelli's theorem provides theoretical basis for a neural net design of $\Theta^{\top} \Phi(x)$ using radial basis functions to keep the approximation error $\varepsilon(x)$ small. ${ }^{19}$

Assumption 2: The set of basis functions $\Phi(x)$ satisfies the persistent excitation (PE) condition for some $\alpha_{0}, \alpha_{1}, T_{0} \geq$ 0

$$
\alpha_{1} I \geq \frac{1}{T_{0}} \int_{t}^{t+T_{0}} \Phi(x(\tau)) \Phi^{\top}(x(\tau)) d \tau \geq \alpha_{0} I, \forall t>0
$$

where $I$ is an identity matrix.

The objective is to design a controller that enables the plant to follow a reference model

$$
\dot{x}_{m}=A_{m} x_{m}+B_{m} r
$$

where $A_{m} \in \mathbb{R}^{n \times n}$ is Hurwitz and known, $B_{m} \in \mathbb{R}^{n \times p}$ is also known, and $r(t):[0, \infty) \rightarrow \mathbb{R}^{p} \in \mathscr{L}_{\infty}$ is a command vector with $\dot{r} \in \mathscr{L}_{\infty}$.

Defining the tracking error as $e=x_{m}-x$, then the controller $u(t)$ is specified by

$$
u=K_{x} x+K_{r} r-u_{a d}
$$


where $K_{x} \in \mathbb{R}^{p \times n}$ and $K_{r} \in \mathbb{R}^{p \times p}$ are known nominal gain matrices, and $u_{a d} \in \mathbb{R}^{p}$ is a direct adaptive signal.

Then, the tracking error equation becomes

$$
\dot{e}=\dot{x}_{m}-\dot{x}=A_{m} e+\left(A_{m}-A-B K_{x}\right) x+\left(B_{m}-B K_{r}\right) r+B\left[u_{a d}-\Theta^{* \top} \Phi(x)-\varepsilon(x)\right]
$$

We choose the gain matrices $K_{x}$ and $K_{r}$ to satisfy the model matching conditions $A+B K_{x}=A_{m}$ and $B K_{r}=B_{m}$. The adaptive signal $u_{a d}$ is an estimator of the parametric uncertainty in the plant such that

$$
u_{a d}=\Theta^{\top} \Phi(x)
$$

where $\Theta \in \mathbb{R}^{m \times p}$ is an estimate of the parametric uncertainty $\Theta^{*}$.

Let $\tilde{\Theta}=\Theta-\Theta^{*}$ be an estimation error of the parametric uncertainty. Then the tracking error equation can be expressed as

$$
\dot{e}=A_{m} e+B\left(\tilde{\Theta}^{\top} \Phi-\varepsilon\right)
$$

Proposition 1: The following adaptive law provides an update law that minimizes $\|e\|_{\mathscr{L}_{2}}$

$$
\dot{\Theta}=-\Gamma \Phi\left(e^{\top} P-v \Phi^{\top} \Theta B^{\top} P A_{m}^{-1}\right) B
$$

where $\Gamma=\Gamma^{\top}>0 \in \mathbb{R}^{m \times m}$ is an adaptive gain matrix, $v>0 \in \mathbb{R}$ is a weighting constant, and $P=P^{\top}>0 \in \mathbb{R}^{n \times n}$ solves

$$
P A_{m}+A_{m}^{\top} P=-Q
$$

where $Q=Q^{\top}>0 \in \mathbb{R}^{n \times n}$.

Proof: The adaptive law seeks to minimize the cost function

$$
J=\frac{1}{2} \int_{0}^{t_{f}}(e-\Delta)^{\top} Q(e-\Delta) d t
$$

subject to Eq. (10) where $\Delta$ represents the tracking error at $t=t_{f}$.

$J$ is convex and represents the distance measured from the normal surface of a ball $B_{r}$ with a radius $\Delta$.

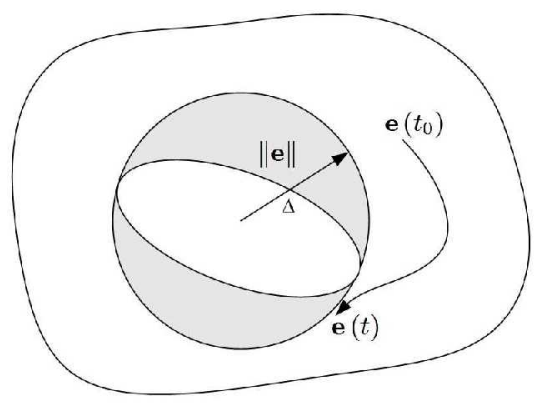

Fig. 2 - Tracking Error Bound

This optimal control problem can be formulated by the Pontryagin's Minimum Principle. Defining a Hamiltonian

$$
H(e, \tilde{\Theta})=\frac{1}{2}(e-\Delta)^{\top} Q(e-\Delta)+p^{\top}\left(A_{m} e+B \tilde{\Theta}^{\top} \Phi-B \varepsilon\right)
$$

where $p(t):[0, \infty) \rightarrow \mathbb{R}^{n}$ is an adjoint variable, then the necessary condition gives

$$
\dot{p}=-\nabla H_{e}^{\top}=-Q(e-\Delta)-A_{m}^{\top} p
$$

with the transversality condition $p\left(t_{f}\right)=0$ since $e(0)$ is known. The optimality condition is obtained by

$$
\nabla H_{\tilde{\Theta}^{\top}}=\Phi \nabla H_{\tilde{\Theta}^{\top} \Phi}=\Phi p^{\top} B
$$


The adaptive law is formulated by the gradient method as

$$
\dot{\tilde{\Theta}}=-\Gamma \nabla H_{\tilde{\Theta}^{\top}}=-\Gamma \Phi p^{\top} B
$$

The solution of $p$ can be obtained using a "sweeping" method ${ }^{20}$ by letting $p=P e+S \Theta^{\top} \Phi$. Then

$$
\dot{P} e+P\left(A_{m} e+B \Theta^{\top} \Phi-B \Theta^{* \top} \Phi-B \varepsilon\right)+\dot{S} \Theta^{\top} \Phi+S \frac{d\left(\Theta^{\top} \Phi\right)}{d t}=-Q(e-\Delta)-A_{m}^{\top}\left(P e+S \Theta^{\top} \Phi\right)
$$

which yields the following equations

$$
\begin{gathered}
\dot{P}+P A_{m}+A_{m}^{\top} P+Q=0 \\
\dot{S}+P B+A_{m}^{\top} S=0 \\
Q \Delta-S \frac{d\left(\Theta^{\top} \Phi\right)}{d t}+P B\left(\Theta^{* \top} \Phi+\varepsilon\right)=0
\end{gathered}
$$

subject to the transversality conditions $P\left(t_{f}\right)=0$ and $S\left(t_{f}\right)=0$.

The existence and uniqueness of the solution of the Lyapunov differential equation (19) is well-established. It follows that Eq. (20) also has a stable, unique solution in time-to-go $\tau=t_{f}-t$.

Since $\dot{r} \in \mathscr{L}_{\infty}, \Phi$ is bounded and Lipschitz, and $p\left(t_{f}\right)=0$ from the transversality condition, then as $t_{f} \rightarrow \infty$, $\lim _{t_{f} \rightarrow \infty}\left|d\left(\Theta^{\top} \Phi\right) / d t\right|$ exists, where

$$
\begin{array}{r}
\lim _{t_{f} \rightarrow \infty}\left|\frac{d\left(\Theta^{\top} \Phi\right)}{d t}\right|=\lim _{t_{f} \rightarrow \infty}\left|-B^{\top} p\left(t_{f}\right) \Phi^{\top} \Gamma \Phi+\Theta^{\top} \frac{\partial \Phi}{\partial x}\left[\dot{x}_{m}-B\left(\tilde{\Theta}^{\top} \Phi-\varepsilon\right)\right]\right| \\
\leq \lim _{t_{f} \rightarrow \infty}\left|\Theta^{\top} L \mathscr{I}\left[-A_{m}^{-1} B_{m} \dot{r}-B\left(\tilde{\Theta}^{\top} \Phi-\varepsilon\right)\right]\right|=\sigma_{t}
\end{array}
$$

for some constant vector $\sigma_{t}>0 \in \mathbb{R}^{n}$, and $\mathscr{I} \in \mathbb{R}^{m \times n}$ is a matrix whose elements are all equal to one.

Consider an infinite time-horizon problem as $t_{f} \rightarrow \infty$, then $P(t) \rightarrow P(0)$ and $S(t) \rightarrow S(0)$. The constant solutions of $P$ and $S$ are determined by their steady state values from Eqs. (19) and (20) that give

$$
\begin{gathered}
P A_{m}+A_{m}^{\top} P=-Q \\
S=-A_{m}^{-\top} P B
\end{gathered}
$$

The adjoint $p$ is then obtained as

$$
p=P e-v A_{m}^{-\top} P B \Theta^{\top} \Phi
$$

where $v$ is introduced as a weighting constant to allow for adjustments of the second term in the adaptive law. Since $\Theta^{*}$ is constant, then the adaptive law (11) is obtained from Eqs. (75) and (25). by

Defining $\delta_{\varepsilon}=\sup _{t}|\varepsilon|$ and $\varphi=\sup _{t}\left|\Theta^{* \top} \Phi\right|$, then for $v=1$ the unknown tracking error $\Delta$ at $t=t_{f} \rightarrow \infty$ is bounded

$$
\|\Delta\| \leq \frac{\lambda_{\max }(P)\|B\|}{\lambda_{\min }(Q)}\left[\|\varphi\|+\left\|\delta_{\varepsilon}\right\|+\frac{\left\|\sigma_{t}\right\|}{\sigma_{\min }\left(A_{m}\right)}\right]
$$

where $\lambda$ and $\sigma$ denote the eigenvalue and singular value, respectively.

Theorem 1: The adaptive law (11) results in stable and uniformly bounded tracking error outside a compact set

$$
\mathscr{W}=\left\{e, \tilde{\Theta}^{\top} \Phi \in \mathbb{R}^{n}: \lambda_{\min }(Q)\left[\left(\|e\|-\Delta_{1}\right)^{2}-\Delta_{1}^{2}\right]+v \lambda_{\min }\left(B^{\top} A_{m}^{-\top} Q A_{m}^{-1} B\right)\left[\left(\left\|\tilde{\Theta}^{\top} \Phi\right\|-\Delta_{2}\right)^{2}-\Delta_{2}^{2}\right] \leq 0\right\}
$$

where

$$
\begin{gathered}
\Delta_{1}=\frac{\lambda_{\max }(P)\|B\|\left\|\delta_{\varepsilon}\right\|}{\lambda_{\min }(Q)} \\
\Delta_{2}=\frac{\sigma_{\max }\left(B^{\top} P A_{m}^{-1} B\right)\|\varphi\|}{\lambda_{\min }\left(B^{\top} A_{m}^{-\top} Q A_{m}^{-1} B\right)} \\
5 \text { of } 20
\end{gathered}
$$


Proof: Choose a Lyapunov candidate function

$$
V=e^{\top} P e+\operatorname{trace}\left(\tilde{\Theta}^{\top} \Gamma^{-1} \tilde{\Theta}\right)
$$

Evaluating $\dot{V}$ yields

$$
\dot{V}=e^{\top}\left(A_{m} P+P A_{m}\right) e+2 e^{\top} P B\left(\tilde{\Theta}^{\top} \Phi-\varepsilon\right)-2 \operatorname{trace}\left[\tilde{\Theta}^{\top} \Phi\left(e^{\top} P B-v \Phi^{\top} \Theta B^{\top} P A_{m}^{-1} B\right)\right]
$$

Using the trace identity trace $\left(A^{\top} B\right)=B A^{\top}, \dot{V}$ can be written as

$$
\dot{V}=-e^{\top} Q e+2 e^{\top} P B\left(\tilde{\Theta}^{\top} \Phi-\varepsilon\right)-2 e^{\top} P B \tilde{\Theta}^{\top} \Phi+2 v \Phi^{\top} \Theta B^{\top} P A_{m}^{-1} B \tilde{\Theta}^{\top} \Phi
$$

The sign-definiteness of the term $P A_{m}^{-1}$ is now considered. We recall that a general real matrix $G$ is positive (negative) definite if and only if its symmetric part $G=\frac{1}{2}\left(G+G^{\top}\right)$ is also positive (negative) definite. Then, by preand post-multiplication of Eq. (12) by $A_{m}^{-\top}$ and $A_{m}^{-1}$, respectively, $P A_{m}^{-1}$ can be decomposed into a symmetric part $M$ and anti-symmetric part $N$ as

$$
P A_{m}^{-1}=M+N
$$

where

$$
\begin{gathered}
M=\frac{1}{2}\left(A_{m}^{-\top} P+P A_{m}^{-1}\right)=-\frac{1}{2} A_{m}^{-\top} Q A_{m}^{-1} \\
N=\frac{1}{2}\left(P A_{m}^{-1}-A_{m}^{-\top} P\right) .
\end{gathered}
$$

Since the symmetric part $M<0$, we conclude that $P A_{m}^{-1}<0$. Then, $\dot{V}$ becomes

$$
\dot{V}=-e^{\top} Q e-2 e^{\top} P B \varepsilon-v \Phi^{\top} \tilde{\Theta} B^{\top} A_{m}^{-\top} Q A_{m}^{-1} B \tilde{\Theta}^{\top} \Phi+2 v \Phi^{\top} \tilde{\Theta} B^{\top} N B \tilde{\Theta}^{\top} \Phi+2 v \Phi^{\top} \Theta^{*} B^{\top} P A_{m}^{-1} B \tilde{\Theta}^{\top} \Phi
$$

Letting $y=B \tilde{\Theta}^{\top} \Phi$ and using the property $y^{\top} N y=0$ for an anti-symmetric matrix $N, \dot{V}$ is reduced to

$$
\dot{V}=-e^{\top} Q e-2 e^{\top} P B \varepsilon-v \Phi^{\top} \tilde{\Theta} B^{\top} A_{m}^{-\top} Q A_{m}^{-1} B \tilde{\Theta}^{\top} \Phi+2 v \Phi^{\top} \Theta^{*} B^{\top} P A_{m}^{-1} B \tilde{\Theta}^{\top} \Phi
$$

which is bounded by

$$
\begin{array}{r}
\dot{V} \leq-\lambda_{\min }(Q)\|e\|^{2}+2\|e\| \lambda_{\max }(P)\|B\|\left\|\delta_{\varepsilon}\right\|-v \lambda_{\min }\left(B^{\top} A_{m}^{-\top} Q A_{m}^{-1} B\right)\left\|\tilde{\Theta}^{\top} \Phi\right\|^{2} \\
+2 v \sigma_{\max }\left(B^{\top} P A_{m}^{-1} B\right)\left\|\Theta^{* \top} \Phi\right\|\left\|\tilde{\Theta}^{\top} \Phi\right\|
\end{array}
$$

By completing the squares, an upper bound of $\dot{V}$ is obtained as

$$
\dot{V} \leq-\lambda_{\min }(Q)\left[\left(\|e\|-\Delta_{1}\right)^{2}-\Delta_{1}^{2}\right]-v \lambda_{\min }\left(B^{\top} A_{m}^{-\top} Q A_{m}^{-1} B\right)\left[\left(\left\|\tilde{\Theta}^{\top} \Phi\right\|-\Delta_{2}\right)^{2}-\Delta_{2}^{2}\right]
$$

If $\mathscr{W}$ is a compact set defined in Eq. (27), then the time rate of change of the Lyapunov candidate function is strictly semi-negative in the complementary set $\mathscr{W}^{c}$ and positive in the compact set $\mathscr{W}$. Thus, the Lyapunov candidate function $V$ decreases everywhere in the complementary set $\mathscr{W}^{c}$, but increases in the compact set $\mathscr{W}$ which contains the origin at $e=0$ and $\tilde{\Theta}=0$. Any trajectory of $e$ and $\tilde{\Theta}$ starting in $\mathscr{W}$ will remain in $\mathscr{W}$ for all $t$. Therefore, the compact set $\mathscr{W}$ is an invariant set. ${ }^{16}$ Then, any trajectory $e$ and $\Theta$ starting in the complementary set $\mathscr{W}^{c}$ will approach the largest invariant set $\mathscr{W}$ as $t \rightarrow \infty .{ }^{21}$ It follows by the LaSalle's Invariance Principle that $e$ and $\Theta$ are ultimately uniformly bounded. Thus, the adaptive optimal control modification is stable.

Remark: The effect of the optimal control modification is to add damping to the weight update law so as to reduce high-frequency oscillations in the weights. The damping term depends on the persistent excitation (PE) condition. With persistent excitation, the weight $\Theta$ is exponentially stable and bounded. This scheme is contrasted to the wellknown $\sigma_{-}{ }^{16}$ and $\varepsilon_{1^{-}}{ }^{18}$ modification methods and other variances which also add damping terms to prevent parameter drift in the absence of the persistent excitation. ${ }^{18}$ These adaptive laws are compared as follows: 


\begin{tabular}{|c|c|}
\hline Modification & Adaptive Law \\
\hline \hline$\sigma-$ & $\dot{\Theta}=-\Gamma\left(\Phi e^{\top} P B+\sigma \Theta\right), \sigma>0$ \\
\hline$\varepsilon_{1-}$ & $\dot{\Theta}=-\Gamma\left(\Phi e^{\top} P B+\mu\left\|e^{\top} P B\right\| \Theta\right), \mu>0$ \\
\hline Optimal & $\dot{\Theta}=-\Gamma\left(\Phi e^{\top} P B-\nu \Phi \Phi^{\top} \Theta B^{\top} P A_{m}^{-1} B\right), v>0$ \\
\hline
\end{tabular}

Table 1 - Modifications to MRAC Law

Theorem 2: In the presence of fast adaptation, i.e., $\lambda_{\min }(\Gamma) \gg 1$, the adaptive law (11) is robustly stable for $v=1$ with all closed-loop poles having negative real values.

Proof: The adaptive law (11) can be written as

$$
\frac{\Phi^{\top} \dot{\Theta}}{\Phi^{\top} \Gamma \Phi}=-\left(e^{\top} P-v \Phi^{\top} \Theta B^{\top} P A_{m}^{-1}\right) B
$$

If $\Gamma \gg 1$ is large and the input is $\mathrm{PE}$, then in the limit as $\Phi^{\top} \Gamma \Phi \rightarrow \infty$

$$
B \Theta^{\top} \Phi \rightarrow \frac{1}{v} P^{-1} A_{m}^{\top} P e
$$

Hence, the closed-loop tracking error equation becomes

$$
\dot{e}=-P^{-1}\left[\left(\frac{1+v}{2 v}\right) Q-\left(\frac{1-v}{2 v}\right) S\right] e-B\left(\Theta^{* \top} \Phi+\varepsilon\right)
$$

where $S=A_{m}^{\top} P-P A_{m}$.

For $v=1$, the closed-loop poles are all real, negative values with $\operatorname{Re}[s]=-\lambda\left(P^{-1} Q\right)$. The system transfer function matrix $H(s)=\left(s I+P^{-1} Q\right)^{-1}$ is strictly positive real (SPR) since $H(j \omega)+H^{\top}(-j \omega)>0$, and thus the system is minimum phase and dissipative. ${ }^{22}$ The Nyquist plot of a strictly stable transfer function for a SISO system is strictly in the right half plane with a phase shift less than or equal to $\frac{\pi}{2},{ }^{22}$ corresponding to a phase margin of at least $\frac{\pi}{2}$. For a MIMO system, the diagonal elements of the system transfer function matrix exhibit a similar behavior.

Lemma 1: The equilibrium state $y=0$ of the differential equation

$$
\dot{y}=-\Phi^{\top}(t) \Gamma \Phi(t) y
$$

where $y(t):[0, \infty) \rightarrow \mathbb{R}, \Phi(t) \in \mathscr{L}_{2}:[0, \infty) \rightarrow \mathbb{R}^{n}$ is a piecewise continuous and bounded function, and $\Gamma>0 \in \mathbb{R}^{n \times n}$, is uniformly asymptotically stable, if there exists a constant $\gamma>0$ such that

$$
\frac{1}{T_{0}} \int_{t}^{t+T_{0}} \Phi^{\top}(\tau) \Gamma \Phi(\tau) d \tau \geq \gamma
$$

which implies that $y$ is locally bounded by the solution of a linear differential equation

$$
\dot{z}=-\gamma z
$$

for $t \in\left[t_{i}, t_{i}+T_{0}\right]$, where $t_{i}=t_{i-1}+T_{0}$ and $i=1,2, \ldots, n \rightarrow \infty$.

Proof: Choose a Lyapunov candidate function and evaluate its time derivative

$$
\begin{gathered}
V=\frac{1}{2} y^{2} \\
\dot{V}=-\Phi^{\top}(t) \Gamma \Phi(t) y^{2}=-2 \Phi^{\top}(t) \Gamma \Phi(t) V
\end{gathered}
$$

Then, there exists $\gamma>0$ for which $V$ is uniformly asymptotically stable since

$$
V\left(t+T_{o}\right)=V(t) \exp \left(-2 \int_{t}^{t+T_{0}} \Phi^{\top}(\tau) \Gamma \Phi(\tau) d \tau\right) \leq V(t) e^{-2 \gamma T_{0}}
$$


This implies that

$$
\exp \left(-2 \int_{t}^{t+T_{0}} \Phi^{\top}(\tau) \Gamma \Phi(\tau) d \tau\right) \leq e^{-2 \gamma T_{0}}
$$

Thus, the equilibrium $y=0$ is uniformly asymptotically stable if

$$
\frac{1}{T_{0}} \int_{t}^{t+T_{0}} \Phi^{\top}(\tau) \Gamma \Phi(\tau) d \tau \geq \gamma
$$

provided $\Phi(t) \in \mathscr{L}_{2}$ is bounded.

Then $y(t) \in \mathscr{L}_{2} \cap \mathscr{L}_{\infty}$ since

$$
V(t \rightarrow \infty)-V(0) \leq-2 \gamma \int_{0}^{\infty} y^{2}(t) d t \Rightarrow 2 \gamma \int_{0}^{\infty} y^{2}(t) d t \leq V(0)-V(t \rightarrow \infty)<\infty
$$

It follows that

$$
\dot{V} \leq-2 \gamma V \Rightarrow y \dot{y} \leq-\gamma y^{2}
$$

which implies that the solution of Eq. (43) is bounded from above if $y \geq 0$ and from below if $y \leq 0$ by the local solution of

$$
\dot{z}=-\gamma z
$$

for $t \in\left[t_{i}, t_{i}+T_{0}\right]$, where $t_{0}=0, t_{i}=t_{i-1}+T_{0}$, and $i=1,2, \ldots, n \rightarrow \infty$.

Now, suppose that $\Phi=\Phi(y)$, Eq. (53) still applies. The condition $\Phi(y(t)) \in \mathscr{L}_{2}$ is identically satisfied since $y \in \mathscr{L}_{2} \cap \mathscr{L}_{\infty}$. To show this, we first evaluate $\dot{V}$ as

$$
\dot{V}=-\Phi^{\top}(y) \Gamma \Phi(y) y^{2}=-2 \Phi^{\top}(y) \Gamma \Phi(y) V
$$

Expressing $V(t)$ as $V(y)$ yields

$$
\dot{V}=\dot{y} \frac{d V}{d y}=-\Phi^{\top}(y) \Gamma \Phi(y) y \frac{d V}{d y}=-2 \Phi^{\top}(y) \Gamma \Phi(y) V
$$

Thus

$$
\frac{d V}{V}=2 \frac{d y}{y}
$$

Suppose we can find $\gamma$ such that

$$
\frac{d y}{y} \leq-\gamma d t
$$

Then multiplying both sides of Eq. (57) by $y^{2}$ and dividing by $d t$ result in the same equation as Eq. (52). Thus, $V$ is uniformly asymptotically stable and $y$ is bounded by the same equation as Eq. (53). Therefore, $\gamma$ given by Eq. (44) satisfies Eq. (57).

Lemma 1 is a version of the well-known Comparison Lemma. ${ }^{21}$ A different version of the proof is also provided by Nadrenda and Annaswamy. ${ }^{23}$

Lemma 2: The solution of a linear differential equation

$$
\dot{y}=A y+g(t)
$$

where $y(t):[0, \infty) \rightarrow \mathbb{R}^{n}, A \in \mathbb{R}^{n \times n}$ is a Hurwitz matrix, and $g(t):[0, \infty) \rightarrow \mathbb{R}^{n} \in \mathscr{L}_{\infty}$ is a piecewise continuous, bounded function, is asymptotically stable and semi-globally bounded from above by the solution of a differential equation

$$
\dot{z}=A\left(z-\alpha\left|A^{-1} c\right|\right)
$$

where $\alpha \geq 1 \in \mathbb{R}$ and $c=\sup _{t}|g(t)|$.

Proof: For matching initial conditions $y(0)=z(0)$, the solutions of $y$ and $z$ are

$$
y=e^{A t} y(0)+\int_{0}^{t} e^{A(t-\tau)} g(\tau) d \tau
$$




$$
z=e^{A t} y(0)-\int_{0}^{t} e^{A(t-\tau)} \alpha A\left|A^{-1} c\right| d \tau
$$

If $A^{-1} c>0$, then

$$
y=z+\int_{0}^{t} e^{A(t-\tau)} \alpha c d \tau+\int_{0}^{t} e^{A(t-\tau)} g(\tau) d \tau=z+\int_{0}^{t} e^{A(t-\tau)} A\left[\alpha A^{-1} c+A^{-1} g(\tau)\right] d \tau
$$

$\alpha \geq 1$ can be made large enough for $\alpha A^{-1} c+A^{-1} g(\tau)>0$ because $A^{-1} c>0$ and $g$ is bounded, and since $\int_{0}^{t} e^{A(t-\tau)} A d \tau \leq 0$, then

$$
\int_{0}^{t} e^{A(t-\tau)} A\left[\alpha A^{-1} c+A^{-1} g(\tau)\right] d \tau \leq 0
$$

Therefore, $y \leq z$.

If $A^{-1} c<0$, then

$$
y=z-\int_{0}^{t} e^{A(t-\tau)} \alpha c d \tau+\int_{0}^{t} e^{A(t-\tau)} g(\tau) d \tau=z-\int_{0}^{t} e^{A(t-\tau)} A\left[\alpha A^{-1} c-A^{-1} g(\tau)\right] d \tau
$$

$\alpha$ can be made large enough for $\alpha A^{-1} c-A^{-1} g(\tau)<0$ because $A^{-1} c<0$ and $g$ is bounded, therefore $y \leq z$. Thus, $y \leq z$ for all $t \in[0, \infty)$ and some $\alpha \geq 1$.

Theorem 3: The steady state tracking error is bounded by

$$
\lim _{t \rightarrow \infty} \sup _{t}\|e\|=\frac{\lambda_{\max }(P)\|B\|}{\sigma_{\min }\left(A_{m}^{\top} P+v P A_{m}\right)}\left[v\|\varphi\|+v\left\|A_{m}\right\|\left\|A_{m}^{-1}\right\|\left\|\delta_{\varepsilon}\right\|+\frac{1}{\gamma}\left\|\left(B^{\top} A_{m}^{-\top} P B\right)^{-1}\right\|\|\beta\|\right]
$$

if there exists a constant $\gamma>0$ such that $\gamma=\inf _{t}\left(\frac{1}{T_{0}} \int_{t}^{t+T_{0}} \Phi^{\top} \Gamma \Phi d \tau\right)>0 \in \mathbb{R}$ and a constant vector $\beta>0 \in \mathbb{R}^{n}$ where $\beta=\sup _{t}\left|\tilde{\Theta}^{\top} \dot{\Phi}\right|$.

Proof: Since $\Theta$ is bounded by the adaptive law (11) and $\lim _{t_{f} \rightarrow \infty}\left|d\left(\Theta^{\top} \Phi\right) / d t\right|<\sigma_{t}$ exists which implies $d\left(\Theta^{\top} \Phi\right) / d t$ is bounded, then $\beta=\sup _{t}\left|\tilde{\Theta}^{\top} \dot{\Phi}\right| \in \mathscr{L}_{\infty}$ is bounded.

Since $e \in \mathscr{L}_{2}, x \in \mathscr{L}_{2}$, and so $\Phi(x) \in \mathscr{L}_{2}$, then using Lemma 1, the adaptive law (11) can be written as

$$
\frac{d}{d t}\left(\tilde{\Theta}^{\top} \Phi\right)=\dot{\tilde{\Theta}}^{\top} \Phi+\tilde{\Theta}^{\top} \dot{\Phi} \leq-\gamma B^{\top} P e+\gamma v B^{\top} A_{m}^{-\top} P B\left(\tilde{\Theta}^{\top} \Phi-\varphi-\left|\left(\gamma v B^{\top} A_{m}^{-\top} P B\right)^{-1} \beta\right|\right)
$$

for $t \in\left[t_{i}, t_{i}+T_{0}\right]$, where $t_{i}=t_{i-1}+T_{0}$ and $i=1,2, \ldots, n \rightarrow \infty$.

Using Lemma 2 with $\alpha=1$ for simplicity, we write

$$
\dot{e} \leq A_{m}\left(e-\left|A_{m}^{-1} B \delta_{\varepsilon}\right|\right)+B \tilde{\Theta}^{\top} \Phi
$$

Differentiating Eq. (67) and upon substitution yields

$$
\begin{aligned}
& \ddot{e}-\left(A_{m}+\gamma v B B^{\top} A_{m}^{-\top} P\right) \dot{e}+\left(\gamma B B^{\top} P+\gamma v B B^{\top} A_{m}^{-\top} P A_{m}\right) e \leq-\gamma v B B^{\top} A_{m}^{-\top} P B\left[\varphi+\left|\left(\gamma v B^{\top} A_{m}^{-\top} P B\right)^{-1} \beta\right|\right] \\
& +\gamma \nu B B^{\top} A_{m}^{-\top} P A_{m}\left|A_{m}^{-1} B \delta_{\varepsilon}\right|
\end{aligned}
$$

whose steady state solution is

$$
\left(A_{m}^{\top} P+v P A_{m}\right) \lim _{t \rightarrow \infty} e \leq-v P B\left[\varphi+\left|\left(\gamma v B^{\top} A_{m}^{-\top} P B\right)^{-1} \beta\right|\right]+v P A_{m}\left|A_{m}^{-1} B \delta_{\varepsilon}\right|
$$

which leads to Eq. (65).

The steady state upper bound on the norm of $\tilde{\Theta}^{\top} \Phi$ is also obtained as

$$
\lim _{t \rightarrow \infty} \sup _{t}\left\|\tilde{\Theta}^{\top} \Phi\right\|=\frac{\lambda_{\max }(P)\left\|A_{m}\right\|}{\sigma_{\min }\left(A_{m}^{\top} P+v P A_{m}\right)}\left[v\|\varphi\|+\left\|A_{m}\right\|\left\|A_{m}^{-1}\right\|\left\|\delta_{\varepsilon}\right\|+\frac{1}{\gamma}\left\|\left(B^{\top} A_{m}^{-\top} P B\right)^{-1}\right\|\|\beta\|\right]
$$


Thus for $\gamma \rightarrow \infty$, the last term on the RHS of Eq. (65) goes to zero, and $\|e\|$ is only dependent on $v$. If, in addition, $v \rightarrow 0$, then $\|e\| \rightarrow 0$, but if $v \rightarrow \infty, e \in \mathscr{L}_{\infty}$ is finite and does not tend to zero. Thus, $v$ has to be selected small enough to provide a desired tracking performance, but large enough to provide sufficient robustness against time delay or unmodeled dynamics. With $v=1$ as the optimal value, further increase the value of $v$ beyond the optimal value will actually reduce robustness as well as tracking performance. Thus, a practical bound for $v$ is $0<v<1$. Both Theorems 2 and 3 provide a guidance in a trade-off design process for selecting a suitable value of $v$ to meet performance and robustness requirements.

\section{Application to Neural Net Adaptive Flight Control}

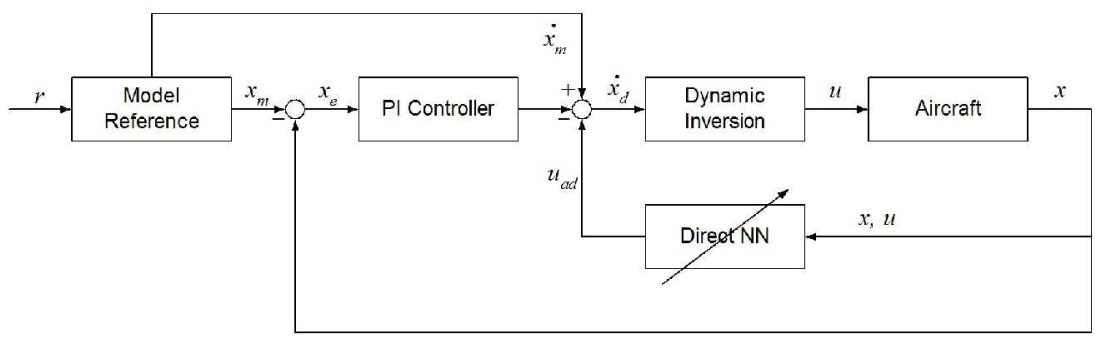

Fig. 3 - Direct Neural Network Adaptive Flight Control

Consider the following inner loop adaptive flight control architecture as shown in Fig. 3. The control architecture comprises: 1) a reference model that translates rate commands into desired acceleration commands, 2) a proportionalintegral (PI) feedback control for rate stabilization and tracking, 3) a dynamic inversion controller that computes actuator commands using desired acceleration commands, and 4) a neural net direct MRAC with the optimal control modification.

Damage adaptive flight control can be used to provide consistent handling qualities and restore stability of aircraft under off-nominal flight conditions such as those due to failures or damage. The linearized equations of motion are expressed as

$$
\begin{aligned}
& \dot{x}=A_{11} x+A_{12} z+B_{1} u+f_{1}(x, z) \\
& \dot{z}=A_{21} x+A_{22} z+B_{2} u+f_{2}(x, z)
\end{aligned}
$$

where $A_{i j}$ and $B_{i}, i=1,2, j=1,2$ are known, $x=\left[\begin{array}{ccc}p & q & r\end{array}\right]^{\top}$ is a vector of roll, pitch, and yaw rates; $z=$ $\left[\begin{array}{cccccc}\Delta \phi & \Delta \alpha & \Delta \beta & \Delta V & \Delta h & \Delta \theta\end{array}\right]^{\top}$ is a vector of perturbation in the bank angle $\Delta \phi$, angle of attack $\Delta \alpha$, sideslip angle $\Delta \beta$, airspeed $\Delta V$, altitude $\Delta h$, and pitch angle $\Delta \theta ; u=\left[\begin{array}{ccc}\Delta \delta_{a} & \Delta \delta_{e} & \Delta \delta_{r}\end{array}\right]^{\top}$ is a vector of additional aileron, elevator, and rudder deflections; and $f_{i}(x, z), i=1,2$ is an uncertainty due to damage which can be approximated as

$$
f_{i}(x, z)=\Theta_{i}^{\top} \Phi(x, z)+\varepsilon
$$

$\Phi(x, z)$ is a basis function for a sigma-pi neural network with $C_{i}, i=1, \ldots, 4$, as inputs consisting of control commands, sensor feedback, and bias terms; defined as follows:

$$
\begin{aligned}
& C_{1}=\frac{1}{2} \rho(h) V^{2}\left[\begin{array}{lll}
x^{\top} & \alpha x^{\top} & \beta x^{\top}
\end{array}\right] \\
& C_{2}=\frac{1}{2} \rho(h) V^{2}\left[\begin{array}{llllllll}
1 & \phi & \theta & \alpha & \beta & \alpha^{2} & \beta^{2} & \alpha \beta
\end{array}\right] \\
& C_{3}=\frac{1}{2} \rho(h) V^{2}\left[\begin{array}{lll}
u(x, z)^{\top} & \alpha u(x, z)^{\top} & \beta u(x, z)^{\top}
\end{array}\right] \\
& C_{4}=\frac{1}{2} \rho(h) V^{2}\left[\begin{array}{lll}
p x^{\top} & q x^{\top} & r x^{\top}
\end{array}\right]
\end{aligned}
$$


where $\phi=\bar{\phi}+\Delta \phi, \alpha=\bar{\alpha}+\Delta \alpha, \beta=\bar{\beta}+\Delta \beta, V=\bar{V}+\Delta V, h=\bar{h}+\Delta h$, and $\theta=\bar{\theta}+\Delta \theta$; and the overbar symbol denotes a trim state.

These inputs are designed to model the unknown nonlinearity that exists in the damaged aircraft plant dynamics. For example, the aerodynamic force in the $x$-axis for an aircraft is given by

$$
\begin{aligned}
F_{x}=T+\frac{1}{2} \bar{q} S\left(C_{L_{0}}+C_{L_{\alpha}} \alpha+C_{L_{\beta}} \beta+C_{L_{p}} \frac{p b}{2 V}+C_{L_{q}} \frac{q \bar{c}}{2 V}+C_{L_{r}} \frac{r b}{2 V}+C_{L_{\delta_{a}}} \delta_{a}+C_{L_{\delta_{e}}} \delta_{e}+C_{L_{\delta_{r}}} \delta_{r}\right) \alpha \\
-\frac{1}{2} \bar{q} S\left(C_{D_{0}}+C_{D_{\alpha}} \alpha+C_{D_{p}} \frac{p b}{2 V}+C_{D_{q}} \frac{q \bar{c}}{2 V}+C_{D_{r}} \frac{r b}{2 V}+C_{D_{\delta_{a}}} \delta_{a}+C_{D_{\delta_{e}}} \delta_{e}+C_{D_{\delta_{r}}} \delta_{r}\right)
\end{aligned}
$$

Thus, $C_{1}, C_{2}$, and $C_{3}$ are designed to model the product terms of $x, z$, and $u$ in the aerodynamic forces and moments equations; and $C_{4}$ models the gyroscopic cross-coupling terms of $x$ in the moment equations.

The inner loop rate feedback control is designed to improve aircraft rate response characteristics such as the short period mode and the dutch roll mode. A second-order reference model is specified to provide desired handling qualities with good damping and natural frequency characteristics as follows:

$$
\begin{aligned}
& \left(s^{2}+2 \zeta_{p} \omega_{p} s+\omega_{p}^{2}\right) \phi_{m}=g_{p} \delta_{l a t} \\
& \left(s^{2}+2 \zeta_{q} \omega_{q} s+\omega_{q}^{2}\right) \theta_{m}=g_{q} \delta_{l o n} \\
& \left(s^{2}+2 \zeta_{r} \omega_{r} s+\omega_{r}^{2}\right) r_{m}=g_{r} \delta_{r u d}
\end{aligned}
$$

where $\phi_{m}, \theta_{m}$, and $\psi_{m}$ are reference bank, pitch, and heading angles; $\omega_{p}, \omega_{q}$, and $\omega_{r}$ are the natural frequencies for desired handling qualities in the roll, pitch, and yaw axes; $\zeta_{p}, \zeta_{q}$, and $\zeta_{r}$ are the desired damping ratios; $\delta_{l a t}, \delta_{l o n}$, and $\delta_{r u d}$ are the lateral stick input, longitudinal stick input, and rudder pedal input; and $g_{p}, g_{q}$, and $g_{r}$ are input gains.

Let $p_{m}=\dot{\phi}_{m}, q_{m}=\dot{\theta}_{m}$, and $r_{m}=\dot{\psi}_{m}$ be the reference roll, pitch, and yaw rates. Then the reference model can be represented as

$$
\dot{x}_{m}=-K_{p} x_{m}-K_{i} \int_{0}^{t} x_{m} d \tau+G r
$$

where $x_{m}=\left[\begin{array}{ccc}p_{m} & q_{m} & r_{m}\end{array}\right]^{\top}, K_{p}=\operatorname{diag}\left(2 \zeta_{p} \omega_{p}, 2 \zeta_{q} \omega_{q}, 2 \zeta_{r} \omega_{r}\right), K_{i}=\operatorname{diag}\left(\omega_{p}^{2}, \omega_{q}^{2}, \omega_{r}^{2}\right), G=\operatorname{diag}\left(g_{p}, g_{q}, g_{r}\right)$, and $r=\left[\begin{array}{lll}\delta_{\text {lat }} & \delta_{\text {lon }} & \delta_{\text {rud }}\end{array}\right]^{\top}$.

Assuming the pair $\left(A_{11}, B_{1}\right)$ is controllable and $z$ is stabilizable, an angular rate feedback dynamic inversion controller is computed as

$$
u_{c}=B_{1}^{-1}\left[-\left(K_{p}+\frac{K_{i}}{s}\right) x+G r-A_{11} x-A_{12} z-\Theta_{1}^{\top} \Phi\right]
$$

where $u_{c}$ is a control surface deflection command vector as an input to a flight control actuator system which can be modeled as a first-order system

$$
u=-\Lambda\left(u-u_{c}\right)
$$

where $\Lambda=\operatorname{diag}\left(\lambda_{a}, \lambda_{e}, \lambda_{r}\right)>0$ is a vector of actuator rates for aileron, elevator, and rudder.

When the actuator dynamics are fast relative to the reference model, i.e., $\lambda_{\min }(\Lambda) \gg \sigma_{\min }\left(A_{m}\right)$, then the actuator output follows the input closely so that $u \approx u_{c}$.

Let $e=\left[\begin{array}{ll}\int_{0}^{t}\left(x_{m}-x\right) d \tau & x_{m}-x\end{array}\right]^{\top}$ be the tracking error, then the tracking error equation is given by

$$
\dot{e}=A_{m} e+B\left(\Theta_{1}^{\top} \Phi-f_{1}\right)
$$

where

$$
\begin{gathered}
A_{m}=\left[\begin{array}{cc}
0 & I \\
-K_{i} & -K_{p}
\end{array}\right] \\
B=\left[\begin{array}{l}
0 \\
I
\end{array}\right]
\end{gathered}
$$


Let $Q=2 I$, then the solution of Eq. (12) yields

$$
P=\left[\begin{array}{cc}
K_{i}^{-1} K_{p}+K_{p}^{-1}\left(K_{i}+I\right) & K_{i}^{-1} \\
K_{i}^{-1} & K_{p}^{-1}\left(I+K_{i}^{-1}\right)
\end{array}\right]>0
$$

$A_{m}^{-1}$ is computed to be

$$
A_{m}^{-1}=\left[\begin{array}{cc}
-K_{i}^{-1} K_{p} & -K_{i}^{-1} \\
I & 0
\end{array}\right]
$$

Evaluating the term $B^{\top} P A_{m}^{-1} B$ yields

$$
B^{\top} P A_{m}^{-1} B=-K_{i}^{-2}<0
$$

Applying the adaptive optimal control modification (11), the weight update law is then given by

$$
\dot{\Theta}_{1}=-\Gamma \Phi\left(e^{\top} P B+v \Phi^{\top} \Theta_{1} K_{i}^{-2}\right)
$$

\section{Simulation Results}

To evaluate the adaptive optimal control modification, a simulation was conducted using a generic transport model (GTM) which represents a notational twin-engine transport aircraft as shown in Fig. $4 .{ }^{24}$ An aerodynamic model of the damaged aircraft is created using a vortex lattice method to estimate aerodynamic coefficients, and stability and control derivatives. For the simulation, a damage configuration is modeled corresponding to a $28 \%$ loss of the left wing. The damage causes an estimated C.G. shift mostly along the pitch axis with $\Delta y=0.0388 \bar{c}$ and an estimated mass loss of $1.2 \%$. The principal moment of inertia about the roll axis is reduced by $12 \%$, while changes in the inertia values in the other two axes are not as significant. Since the damaged aircraft is asymmetric, the inertia tensor has all six non-zero elements. This means that all the three roll, pitch, and yaw axes are coupled together throughout the flight regime.

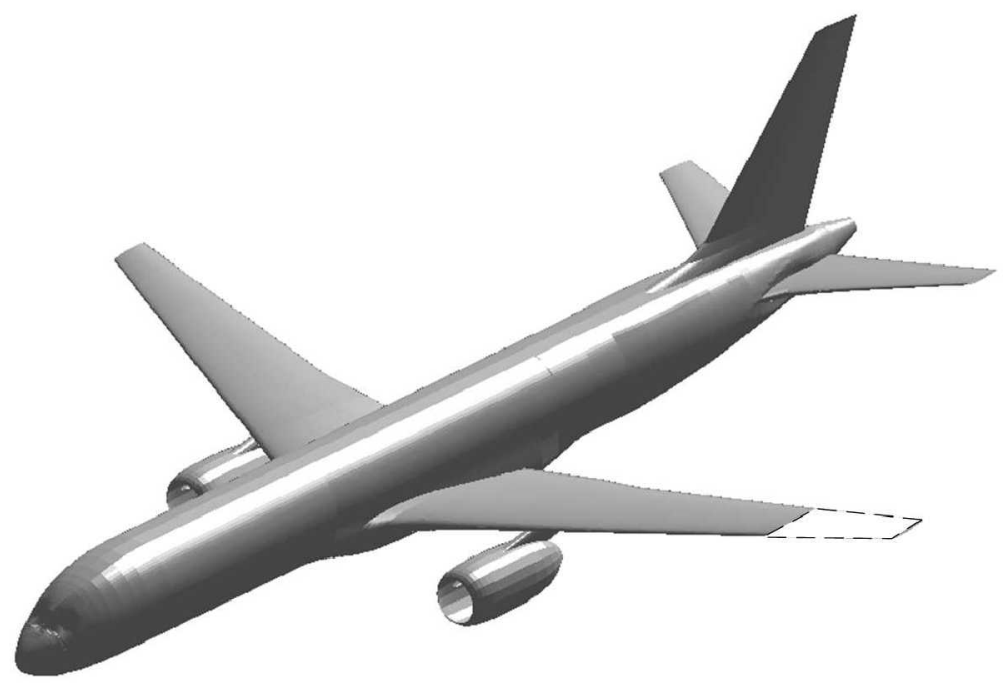

Fig. 4 - Left Wing Damaged Generic Transport Model

A level flight condition of Mach 0.6 at $15,000 \mathrm{ft}$ is selected. Upon damage, the aircraft is re-trimmed with $T=$ $13,951 \mathrm{lb}, \bar{\alpha}=5.86^{\circ}, \bar{\phi}=-3.16^{\circ}, \bar{\delta}_{a}=27.32^{\circ}, \bar{\delta}_{e}=-0.53^{\circ}, \bar{\delta}_{r}=-1.26^{\circ}$. The remaining right aileron is the only roll control effector available. In practice, some aircraft can control a roll motion with spoilers, which are not modeled in this study. The reference model is specified by $\omega_{p}=2.0 \mathrm{rad} / \mathrm{sec}, \omega_{q}=1.5 \mathrm{rad} / \mathrm{sec}, \omega_{r}=1.0 \mathrm{rad} / \mathrm{sec}$, and $\zeta_{p}=\zeta_{q}=\zeta_{r}=1 / \sqrt{2}$. 
The state space model of the damaged aircraft is given by

$$
\begin{aligned}
{\left[\begin{array}{c}
\dot{p} \\
\dot{q} \\
\dot{r}
\end{array}\right]=\left[\begin{array}{cccc}
-1.3623 & -0.2649 & 0.5470 \\
-0.0659 & -0.8949 & 0.0151 \\
0.0835 & -0.00417 & -0.5130
\end{array}\right]\left[\begin{array}{l}
p \\
q \\
r
\end{array}\right] } \\
+\left[\begin{array}{cccccc}
0 & -10.9768 & -9.2449 & -0.6380 & 0.0004 & 0 \\
-0.0007 & -2.7042 & -0.0120 & -0.1409 & -0.0004 & 0 \\
0 & 0.1845 & 2.8755 & -0.0149 & 0 & 0
\end{array}\right]\left[\begin{array}{c}
\Delta \phi \\
\Delta \alpha \\
\Delta \beta \\
\Delta V / \bar{V} \\
\Delta h / \bar{h} \\
\Delta \theta
\end{array}\right] \\
+\left[\begin{array}{ccc}
3.2225 & -0.0449 & 1.4887 \\
0.3393 & -3.4650 & 0.0258 \\
-0.0123 & 0.0007 & -2.2949
\end{array}\right]\left[\begin{array}{c}
\Delta \delta_{a} \\
\Delta \delta_{e} \\
\Delta \delta_{r}
\end{array}\right]
\end{aligned}
$$

$$
\begin{aligned}
& {\left[\begin{array}{c}
\Delta \dot{\phi} \\
\Delta \dot{\alpha} \\
\Delta \dot{\beta} \\
\Delta \dot{V} / \bar{V} \\
\Delta \dot{h} / \bar{h} \\
\Delta \dot{\theta}
\end{array}\right]=\left[\begin{array}{ccc}
1 & 0 & 0.1024 \\
-0.0059 & 0.9722 & 0.0004 \\
0.0978 & -0.0001 & -0.9849 \\
0.0001 & -0.0014 & -0.0006 \\
0 & 0 & 0 \\
0 & 1 & 0.0551
\end{array}\right]\left[\begin{array}{l}
p \\
q \\
r
\end{array}\right]} \\
& +\left[\begin{array}{cccccc}
0 & 0 & 0 & 0 & 0 & 0 \\
0.0028 & -0.4802 & 0.0238 & -0.0648 & 0.0304 & 0 \\
0.0507 & 0.0016 & -0.1751 & -0.1850 & 0.0028 & -0.0017 \\
0 & -0.0453 & 0.0044 & -0.0082 & 0 & -0.0507 \\
0 & -0.0423 & 0 & 0 & 0 & 0.0423 \\
0 & 0 & 0 & 0 & 0 & 0
\end{array}\right]\left[\begin{array}{c}
\Delta \phi \\
\Delta \alpha \\
\Delta \beta \\
\Delta V / \bar{V} \\
\Delta h / \bar{h} \\
\Delta \theta
\end{array}\right] \\
& +\left[\begin{array}{ccc}
0 & 0 & 0 \\
0.0241 & -0.0704 & -0.0012 \\
0.0053 & 0 & 0.0604 \\
0.0018 & -0.0048 & -0.0028 \\
0 & 0 & 0 \\
0 & 0 & 0
\end{array}\right]\left[\begin{array}{c}
\Delta \delta_{a} \\
\Delta \delta_{e} \\
\Delta \delta_{r}
\end{array}\right]
\end{aligned}
$$

The actuator dynamics are modeled with $\lambda_{a}=\lambda_{e}=\lambda_{r}=50 / \mathrm{sec}$ with position limits of $\pm 35^{\circ}$ for the aileron and elevator and $\pm 10^{\circ}$ for the rudder.

The pilot pitch rate command is simulated with a series of ramp input longitudinal stick command doublets, corresponding to the reference pitch angle $\pm 3.81^{\circ}$ from trim. The tracking performance of the baseline flight control with no adaptation is compared against the neural net direct MRAC, the $\varepsilon_{1}$ modification with $\mu=0.1$, and the present adaptive optimal control modification with $v=0.1$. The adaptive gains are selected as large as possible within the numerical stability limit of the adaptive laws. This results in $\Gamma=60$ for direct MRAC, $\Gamma=800$ for the $\varepsilon_{1}$ modification, and $\Gamma=2580$ for the adaptive optimal control modification. Thus, it can be seen that the optimal control modification can tolerate a much larger adaptive gain than the standard MRAC. This large adaptive gain allows the adaptive optimal control modification to better adapt to uncertainties than the standard direct MRAC. 

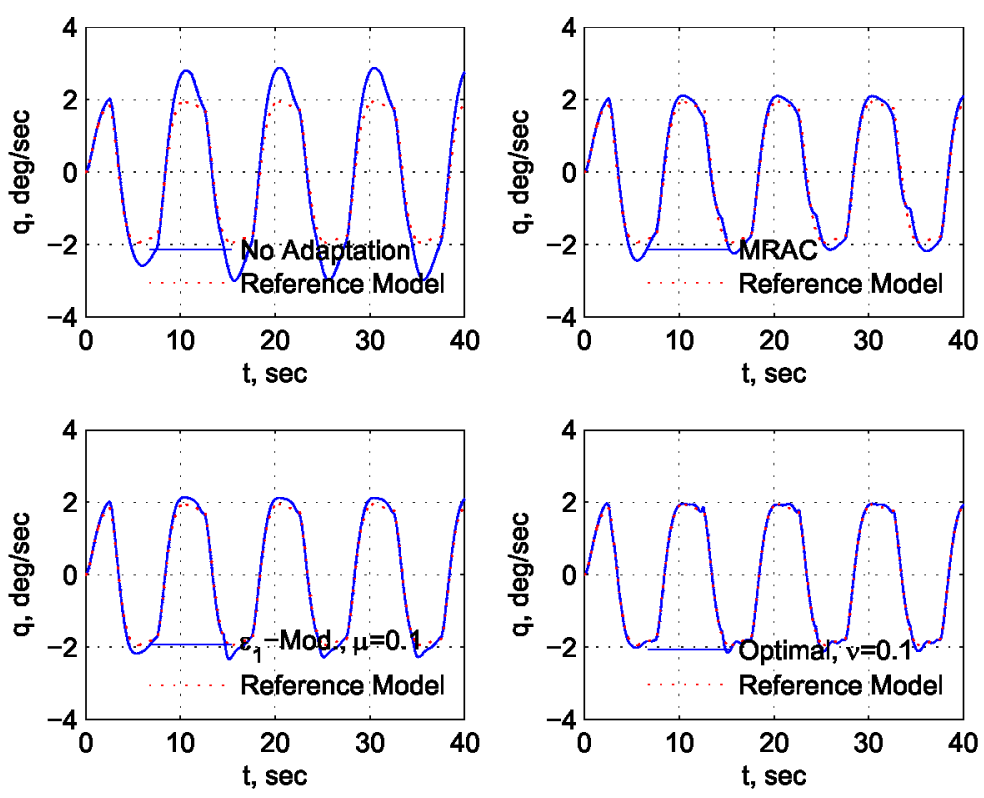

Fig. 5 - Pitch Rate

The aircraft angular rate responses are shown in Figs. 5 to 8. Figure 5 illustrates the pitch rate response due to the four controllers. With no adaptation, the baseline flight control system cannot follow the reference pitch rate very well as there is a significant overshoot. Both the direct MRAC and the $\varepsilon_{1}$ modification improves the tracking by about the same amount. However, the adaptive optimal control modification appears to provide better tracking than the MRAC and the $\varepsilon_{1}$ modification.

Since the damage occurs to one of the wings, the roll axis is most affected. With no adaptation, there is a significant roll rate as high as $20^{\circ} / \mathrm{sec}$ as shown in Fig. 6. Both the direct MRAC and $\varepsilon_{1}$ modification reduce to maximum amplitude of the roll rate to about $10^{\circ} / \mathrm{sec}$. The adaptive optimal control modification further reduces the roll rate to a maximum value of about $4 \%$ sec.
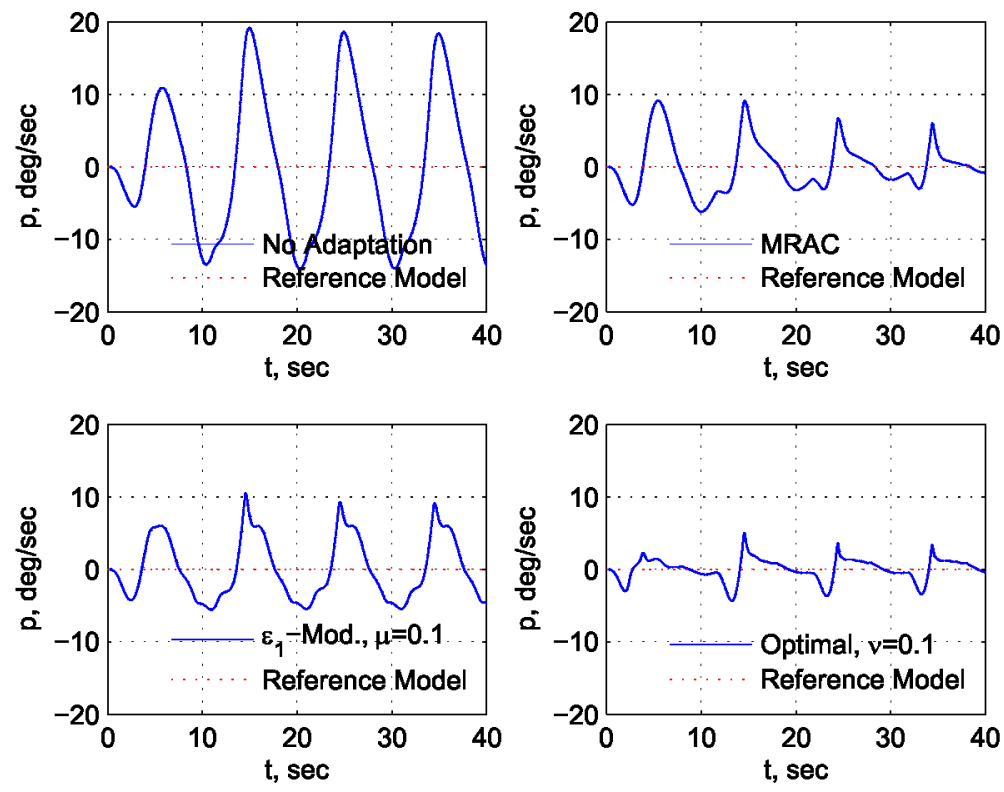

Fig. 6 - Roll Rate

Figure 7 is the yaw rate response of the damaged aircraft. All the three adaptive controllers significantly reduce the roll rate to a reasonably low level. The $\varepsilon_{1}$ modification performs slightly better than the MRAC and adaptive optimal control modification. 

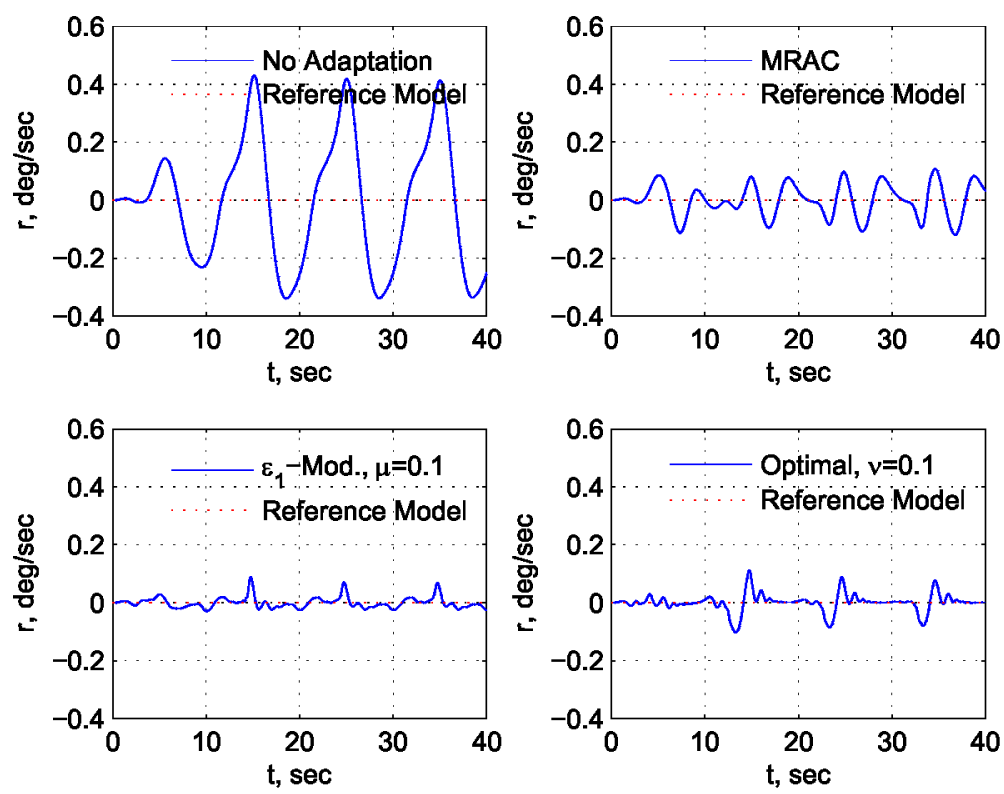

Fig. 7 - Yaw Rate

Figure 8 is the plot of the tracking error $\mathscr{L}_{2}$ norm for all the three aircraft angular rates that compares the overall performance of the four controllers collectively in the three axes. When there is no adaptation, the tracking error norm appears to grow considerably in the first $10 \mathrm{sec}$. The $\varepsilon_{1}$ modification actually results in higher tracking error than the direct MRAC. This could be explained by the fact that the $\varepsilon_{1}$ modification trades performance for robustness, so the tracking performance is expected to be worse. The adaptive optimal control modification results in the smallest tracking error norm as compared to the MRAC and $\varepsilon_{1}$ modification. Thus, overall, the adaptive optimal control modification performs significantly better than the MRAC and $\varepsilon_{1}$ modification.
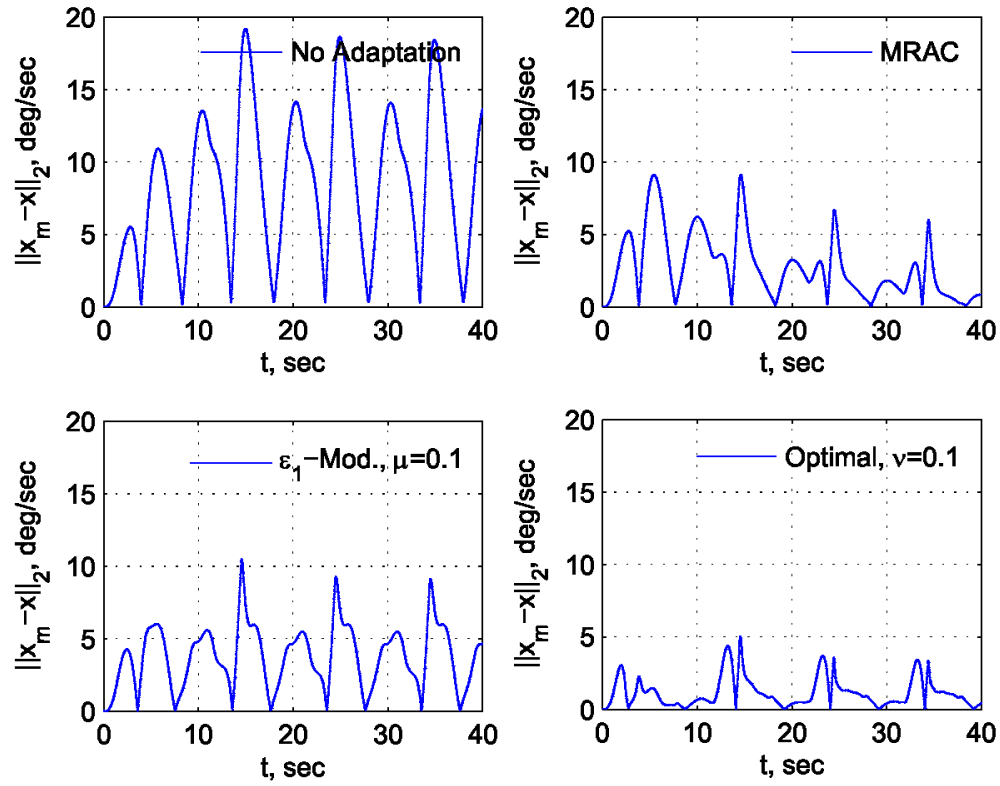

Fig. 8 - Tracking Error Norm

The attitude responses of the damaged aircraft are shown in Figs. 9 to 12 . When there is no adaptation, the pitch attitude could not be followed accurately as seen in Fig. 9. With adaptation on, the tracking is much improved and the adaptive optimal control modification follows the pitch command better than the direct MRAC and the $\varepsilon_{1}$ modification. 

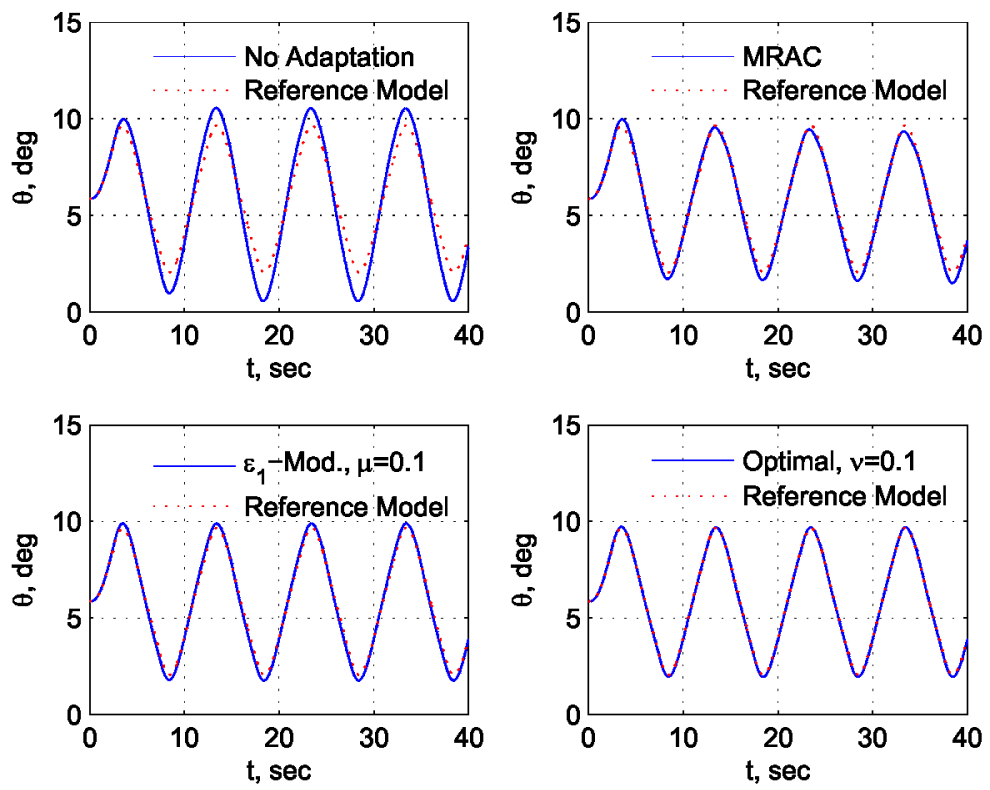

Fig. 9 - Pitch Angle

Figure 10 is the plot of the bank angle. Without adaptation, the damaged aircraft exhibits a rather severe roll behavior with the bank angle ranging from $-30^{\circ}$ to $20^{\circ}$. Both the direct MRAC and $\varepsilon_{1}$ modification improve the situation significantly. The adaptive optimal control shows a drastic improvement in the arrest of the roll motion with the bank angle maintained closed to the trim value.
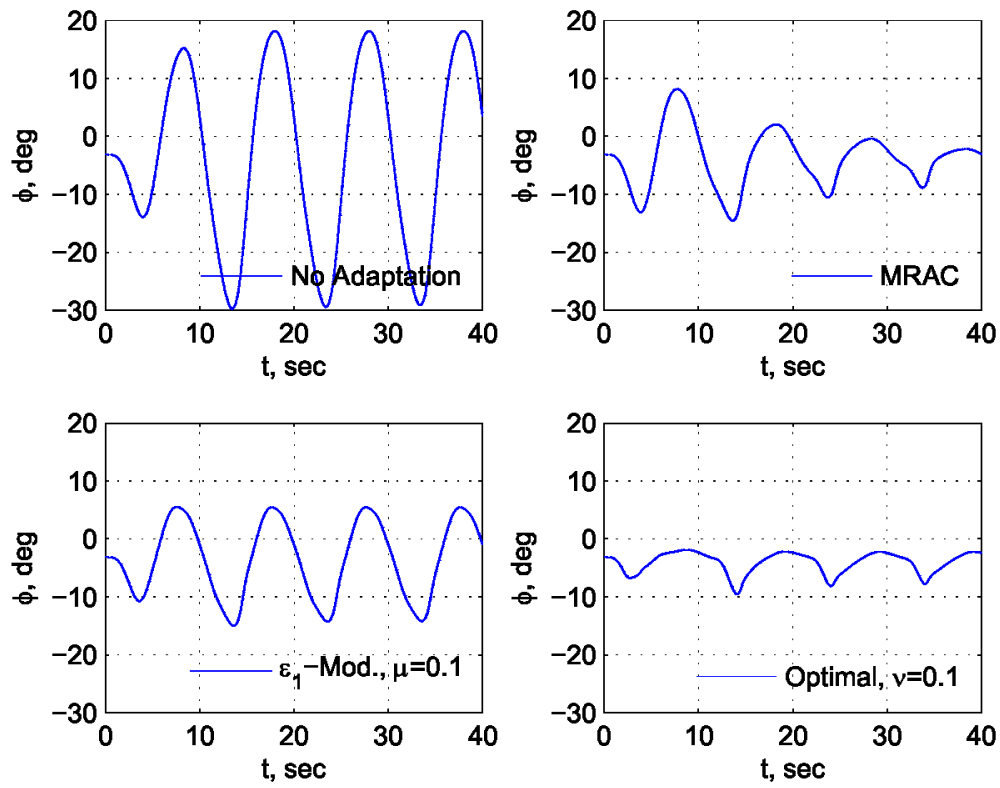

Fig. 10 - Bank Angle

Figure 11 is a plot of the angle of attack. All the adaptive controllers produce similar angle of attack responses. 

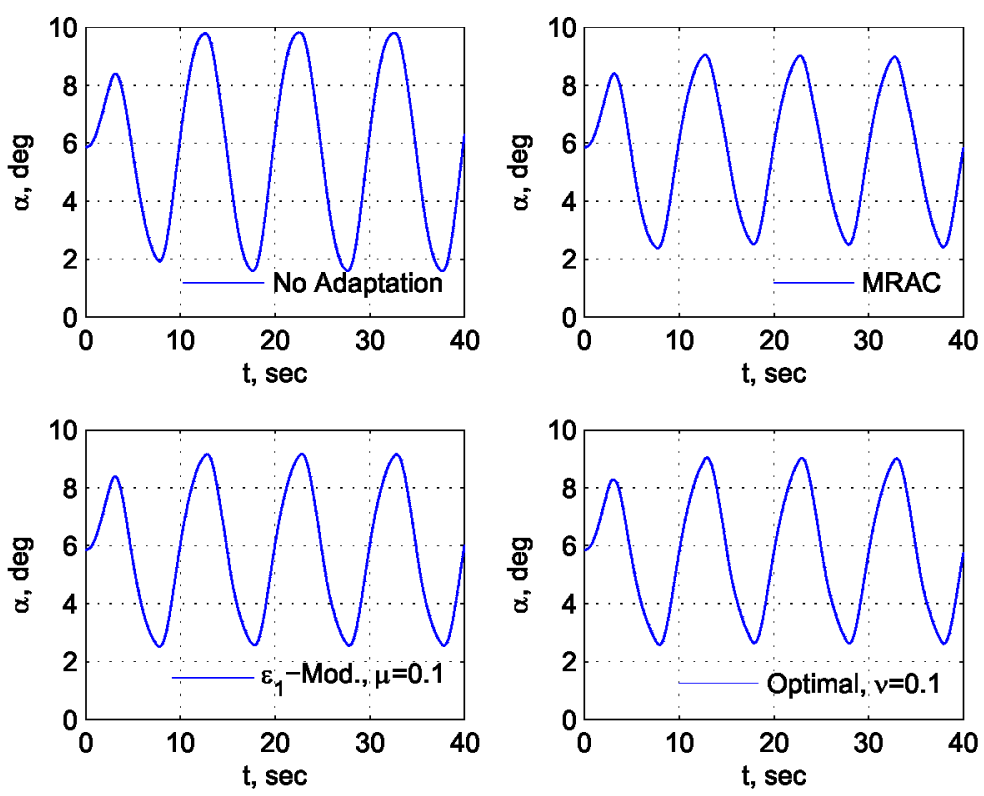

Fig. 11 - Angle of Attack

Figure 12 shows a plot of the sideslip angle. In general, flying with sideslip angle is not a common practice since a large sideslip angle can cause an increase in drag and more importantly a decrease in the yaw damping. With the adaptive optimal control modification, the sideslip angle is reduced to near zero, while the direct MRAC and $\varepsilon_{1}$ modification still show some sideslip angle responses.
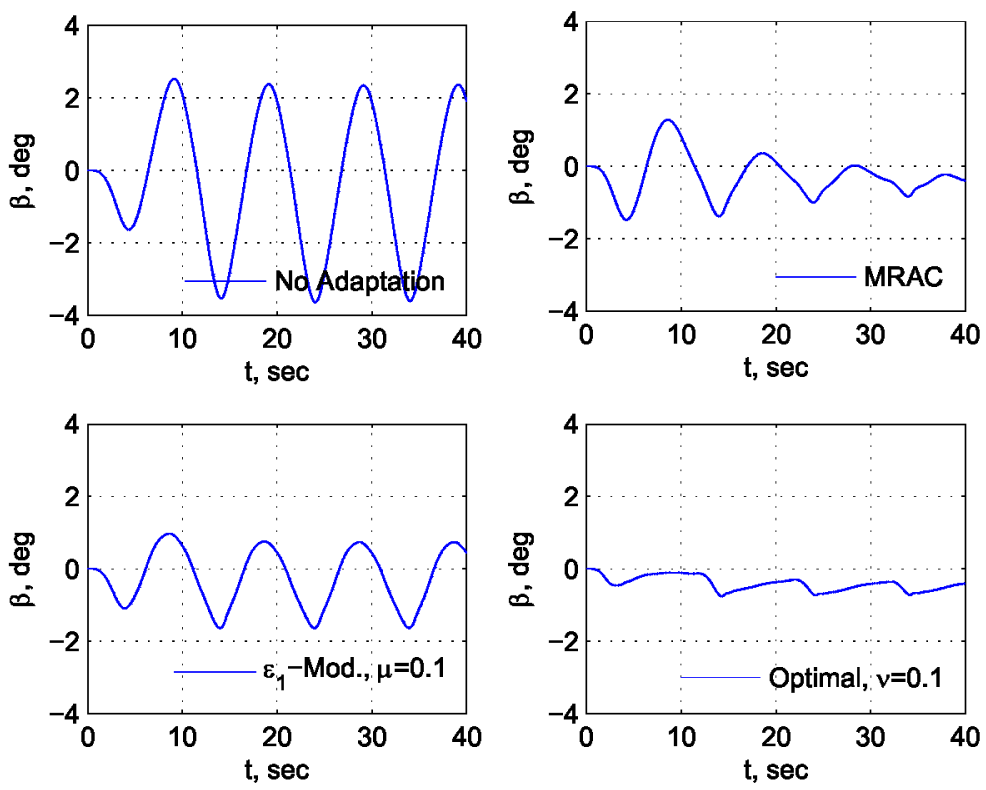

Fig. 12 - Sideslip Angle

The control surface deflections are plotted in Figs. 10 to 12. Because of the wing damage, the damaged aircraft has to be trimmed with a rather large aileron deflection. This causes the roll control authority to severely decrease. Any pitch maneuver can potentially run into a control saturation in the roll axis due to the pitch-roll coupling that exists in a wing damage scenario. With the maximum aileron deflection at $35^{\circ}$, it can be seen clearly from Fig. 13 that a roll control saturation is present in all cases. The range of aileron deflection when there is no adaptation is quite large. As the aileron deflection hits the maximum position limit, it tends to over-compensate in the down swing because of the large pitch rate error produced by the control saturation. 

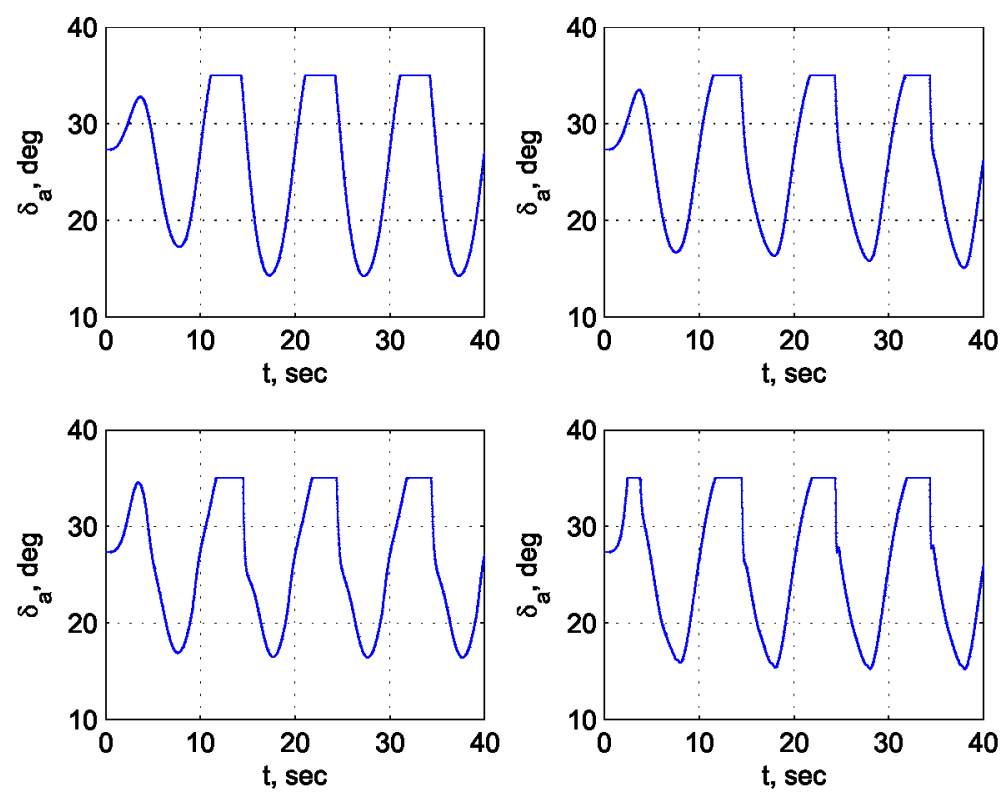

Fig. 13 - Aileron Deflection

Figure 14 is a plot of the elevator deflection which is shown to be within a range of few degrees for all the four controllers and well within the control authority of the elevator. This implies that the roll control contributes mostly to the overall response of the wing-damaged aircraft.
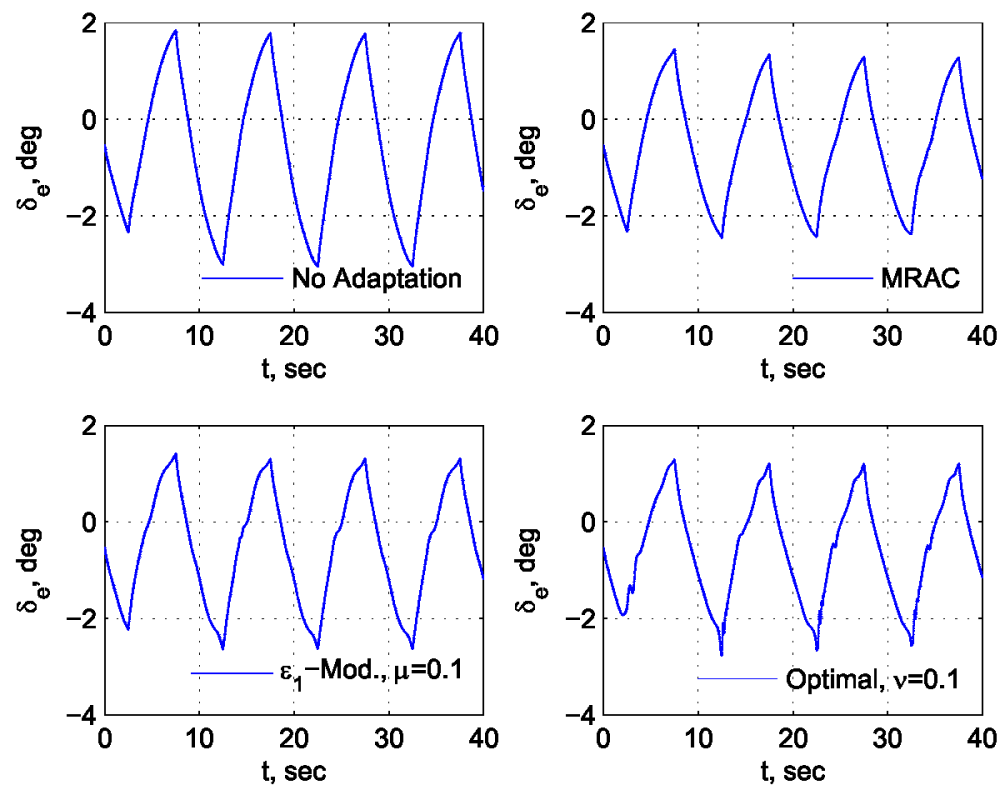

Fig. 14 - Elevator Deflection

The rudder deflection is shown in Fig. 15. With no adaptation, the rudder deflection is quite active, going from almost $-7^{\circ}$ to $2^{\circ}$. While this appears small, it should be compared relative to the rudder position limit, which is usually reduced as the airspeed and altitude increase. The absolute rudder position limit is $\pm 10^{\circ}$ but in practice the actual rudder position limit may be less. Therefore, it is usually desired to keep the rudder deflection as small as possible. Both the direct MRAC and $\varepsilon_{1}$ modification improve the situation somewhat, but the adaptive optimal control is able to keep the rudder deflection quite small and virtually almost at trim. 

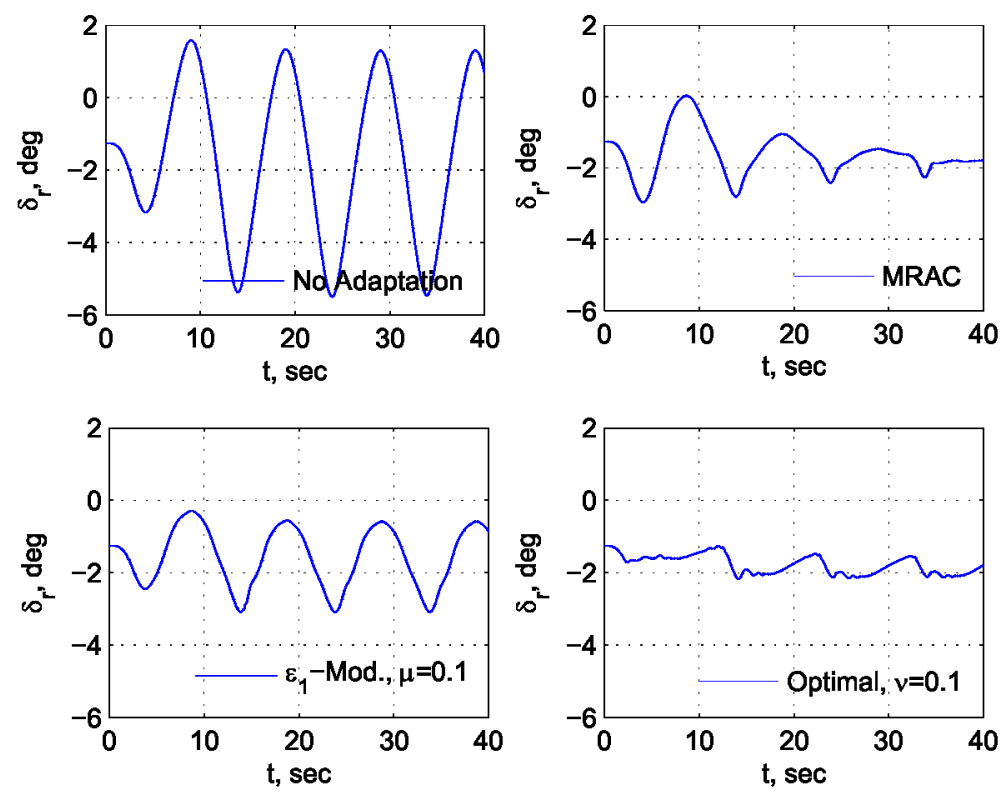

Fig. 15 - Rudder Deflection

To demonstrate stability robustness of the adaptive optimal control modification, the time delay margin (TDM) is computed numerically in the simulations as a function of the parameter $v$. A time delay is introduced between the actuators and the damaged aircraft plant model and is adjusted until the adaptive optimal control modification algorithm is on the verge of instability. The results are plotted in Fig. 16 for an adaptive gain $\Gamma=60$. As $v$ increases, the time delay margin also increases. This results in a more robust controller that can tolerate a larger time delay which acts as a destabilizing disturbance to the controller. However, for the same adaptive gain, increasing $v$ tends to degrade the tracking performance. Therefore, in general, $v$ is selected to balance the competing requirements for performance and stability robustness that usually exist in a control design.

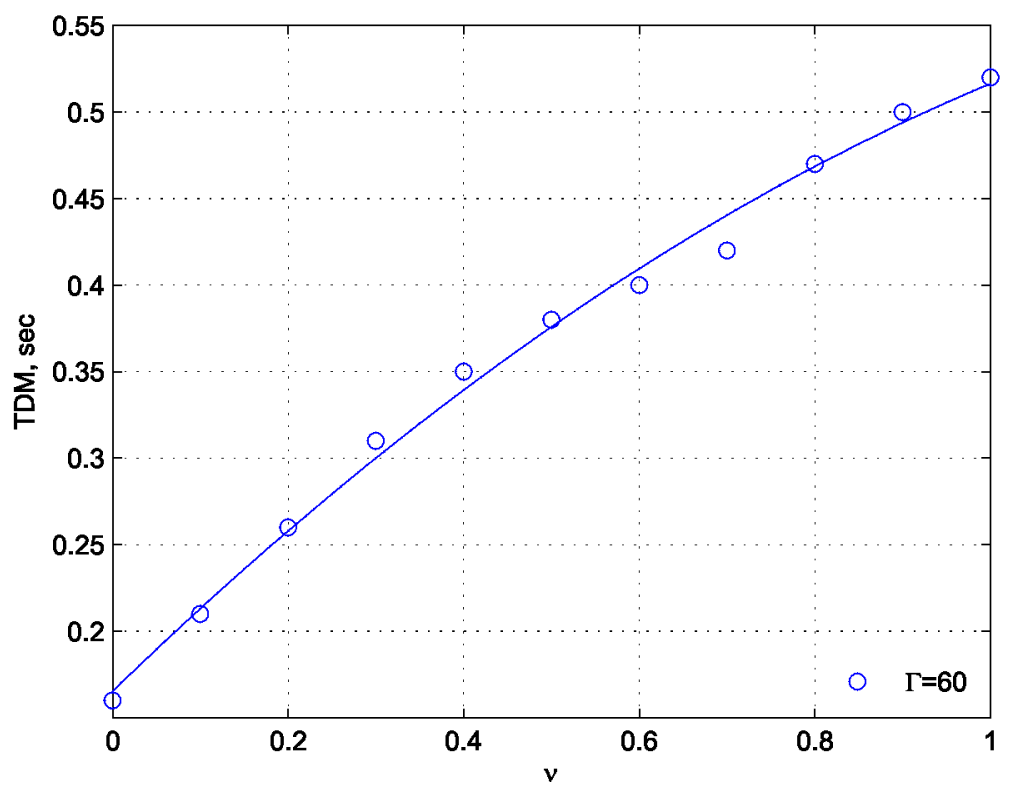

Fig. 16 - Estimated Time Delay Margin

\section{Conclusions}

This study presents a new modification to the standard model-reference adaptive control based on an optimal control formulation of minimizing the $\mathscr{L}_{2}$ norm of the tracking error. The adaptive optimal control modification adds 
a damping term to the adaptive law that is proportional to the persistent excitation. The modification enables fast adaptation without sacrificing robustness. The modification can be tuned using a parameter $v$ to provide a trade-off between tracking performance and stability robustness. Increasing $v$ results in better stability margins but reduced tracking performance. When $v$ approaches unity, the system is robustly stable with all closed-loop poles having negative real values. Simulations of a damaged generic transport aircraft were conducted. The results demonstrate the effectiveness of the adaptive optimal modification, which shows that tracking performance can be achieved at a much larger adaptive gain than the standard direct model-reference adaptive control. As a result, significant improvements in performance can be attained with the adaptive optimal control modification.

\section{References}

\footnotetext{
${ }^{1}$ Bosworth, J. and Williams-Hayes, P.S., "Flight Test Results from the NF-15B IFCS Project with Adaptation to a Simulated Stabilator Failure", AIAA Infotech@Aerospace Conference, AIAA-2007-2818, 2007.

${ }^{2}$ Sharma, M., Lavretsky, E., and Wise, K., "Application and Flight Testing of an Adaptive Autopilot On Precision Guided Munitions", AIAA Guidance, Navigation, and Control Conference, AIAA-2006-6568, 2006.

${ }^{3}$ Rohrs, C.E., Valavani, L., Athans, M., and Stein, G., "Robustness of Continuous-Time Adaptive Control Algorithms in the Presence of Unmodeled Dynamics", IEEE Transactions on Automatic Control, Vol AC-30, No. 9, pp. 881-889, 1985.

${ }^{4}$ Nguyen, N., and Jacklin, S., "Neural Net Adaptive Flight Control Stability, Verification and Validation Challenges, and Future Research", Workshop on "Applications of Neural Networks in High Assurance Systems", International Joint Conference on Neural Networks, 2007.

${ }^{5}$ Jacklin, S. A., Schumann, J. M., Gupta, P. P., Richard, R., Guenther, K., and Soares, F., "Development of Advanced Verification and Validation Procedures and Tools for the Certification of Learning Systems in Aerospace Applications", AIAA Infotech@Aerospace Conference, AIAA-2005-6912, 2005.

${ }^{6}$ Steinberg, M. L., "A Comparison of Intelligent, Adaptive, and Nonlinear Flight Control Laws", AIAA Guidance, Navigation, and Control Conference, AIAA-1999-4044, 1999.

${ }^{7}$ Eberhart, R. L. and Ward, D. G., "Indirect Adaptive Flight Control System Interactions", International Journal of Robust and Nonlinear Control, Vol. 9, pp. 1013-1031, 1999.

${ }^{8}$ Rysdyk, R. T. and Calise, A. J., "Fault Tolerant Flight Control via Adaptive Neural Network Augmentation", AIAA Guidance, Navigation, and Control Conference, AIAA-1998-4483, 1998.

${ }^{9}$ Kim, B. S. and Calise, A. J., "Nonlinear Flight Control Using Neural Networks", Journal of Guidance, Control, and Dynamics, Vol. 20 , No. 1, pp. 26-33, 1997.

${ }^{10}$ Johnson, E. N., Calise, A. J., El-Shirbiny, H. A., and Rysdyk, R. T., "Feedback Linearization with Neural Network Augmentation Applied to X-33 Attitude Control", AIAA Guidance, Navigation, and Control Conference, AIAA-2000-4157, 2000.

${ }^{11}$ Idan, M., Johnson, M. D., and Calise, A. J., "A Hierarchical Approach to Adaptive Control for Improved Flight Safety", AIAA Journal of Guidance, Control and Dynamics, Vol. 25, No. 6, pp. 1012-1020, 2002.

${ }^{12}$ Hovakimyan, N., Kim, N., Calise, A. J., Prasad, J. V. R., and Corban, E. J., "Adaptive Output Feedback for High-Bandwidth Control of an Unmanned Helicopter", AIAA Guidance, Navigation and Control Conference, AIAA-2001-4181, 2001.

${ }^{13}$ Cao, C. and Hovakimyan, N., "Design and Analysis of a Novel L1 Adaptive Control Architecture with Guaranteed Transient Performance", IEEE Transactions on Automatic Control, Vol. 53, No. 2, pp. 586-591, 2008.

${ }^{14}$ N. Nguyen, K. Krishnakumar, J. Kaneshige, and P. Nespeca, "Flight Dynamics and Hybrid Adaptive Control of Damaged Aircraft", AIAA Journal of Guidance, Control, and Dynamics, Vol. 31, No. 3, pp. :751-764, 2008.

${ }^{15}$ Nguyen, N., Krishnakumar, K., and Boskovic, J., "An Optimal Control Modification to Model-Reference Adaptive Control for Fast Adaptation", AIAA Guidance, Navigation, and Control Conference, AIAA 2008-7283, 2008.

${ }^{16}$ Ioannu, P.A. and Sun, J., Robust Adaptive Control, Prentice-Hall, 1996. 1989.

${ }^{17}$ Cybenko, G., "Approximation by Superpositions of a Sigmoidal Function", Mathematics of Control Signals Systems, Vol. 2, pp. 303-314,

${ }^{18}$ Narendra, K. S. and Annaswamy, A. M., "A New Adaptive Law for Robust Adaptation Without Persistent Excitation", IEEE Transactions on Automatic Control, Vol. AC-32, No. 2, pp. 134-145, 1987.

${ }^{19}$ Micchelli, C. A., "Interpolation of Scattered Data: Distance Matrices and Conditionally Positive Definite Functions", Constructive Approximation, Vol.2, pp. 11-12, 1986.

${ }^{20}$ Bryson, A.E. and Ho, Y.C., Applied Optimal Control: Optimization, Estimation, and Control, John Wiley \& Sons Inc., 1979.

${ }^{21}$ Khalil, H. K., Nonlinear Systems, Prentice-Hall, 2002.

${ }^{22}$ Slotine, J.-J. and Li, W., Applied Nonlinear Control, Prentice-Hall, 1991.

${ }^{23}$ Narendra, K.S. and Annaswamy, A.M., Stable Adaptive Systems, Dover Publications, 2005.

${ }^{24}$ Bailey, R. M, Hostetler, R. W., Barnes, K. N., Belcastro, C. M. and Belcastro, C. M., "Experimental Validation: Subscale Aircraft Ground Facilities and Integrated Test Capability", AIAA Guidance, Navigation, and Control Conference, AIAA-2005-6433, 2005.
} 